\title{
La PARIDAD DE GÉNERO EN LAS ELECCIONES LOCALES 2015 DE TABASCO, Estado de MÉXico y Distrito Federal
}

\author{
Gender Parity in 2015 Local Elections in Tabasco, State of Mexico and Mexico City \\ Rafael Cedillo-Delgado
}

Resumen: En este artículo se examina el cumplimiento y efectividad de la cuota de género en tres entidades donde se efectuaron elecciones locales en 2015: Tabasco, Distrito Federal y Estado de México. A propósito de la reciente incorporación de la paridad de género, que exige a los partidos políticos incluir el 50\% de candidatos de cada sexo, aquí se ilustran las prácticas e interpretaciones que hacen los partidos políticos y órganos electorales en la aplicación de dicha medida. Se destaca que en Tabasco se logró la paridad en la integración del Congreso local, en contraste con lo ocurrido en el Distrito Federal y en el Estado de México.

Palabras clave: sistema electoral, elecciones, participación política, mujeres, igualdad de oportunidades.

Abstract: This article examines the degree of accomplishment and effectiveness of the gender quota in three states where local elections were held in 2015: Tabasco, Mexico City, and State of Mexico. The article looks at the new incorporation of gender parity, wich force political parties to include candidatures of $50 \%$ candidates for each sex, illustrates how this measure is interpreted and put in practice by political parties and electoral bodies. The article shows that in Tabasco parity was achieved in the integration of the Local Congress, in contrast to what happened in the Federal District and State of Mexico.

Keywords: electoral system, elections, political participation, women, equal opportunity.

Rafael Cedillo Delgado, doctor en Ciencias Sociales por la Universidad AutónomadelEstadode México. Maestroen Estudios Latinoamericanos por la Universidad Nacional Autónoma de México. Profesor de tiempo completo en la Universidad Autónoma del Estado de México, campus Amecameca y Texcoco, México. Temas de especialización: diversidad y cuotas electorales. Correo electrónico: rafa_cd2000@yahoo.com.mx.
Enviado a dictamen: 14 de agosto de 2015.

Aprobación: 30 de septiembre de 2015.

Revisiones: 1. 


\section{Introducción}

$\mathrm{D}$ e cara al proceso electoral federal de 2015, en donde se renovaron los quinientos miembros de la Cámara de Diputados, y en diecisiete estados donde se efectuaran elecciones locales para renovar los congresos locales y elegir nueve gobernadores, se puso en marcha la recién aprobada cuota de género, que establece el principio de paridad entre hombres y mujeres para tener acceso a cargos de elección popular. Dicha innovación, junto con las candidaturas independientes, llamó la atención por el impacto que tendría en la vida política mexicana.

La Ley General de Instituciones y Procedimientos Electorales (LEGIPE), aprobada en mayo de 2014, establece en sus artículos 7, 232, 233, 234 y 235 la obligatoriedad de que los partidos políticos garanticen la paridad entre los géneros en la postulación de candidatos a los cargos de elección para la integración del Congreso de la Unión, los Congresos de los Estados y la Asamblea Legislativa del Distrito Federal (Carbonell, 2015: 155-157). Además se especificó que debe respetarse el principio de paridad en el principio de representación proporcional, en el orden y en las fórmulas.

Si bien es cierto que en algunos países de América Latina ya se ha establecido la cuota de género bajo el principio de paridad, como en Bolivia (2009), Costa Rica (2009), Ecuador (2008), Honduras (2012) y Nicaragua (2010), lo cierto es que la inclusión legal de dicha medida no garantiza el equilibrio en la representación política. Estudios y voces al respecto han manifestado que hay vacíos legales, y que muchas prácticas de los partidos políticos propician que no se logre el cumplimiento, por lo que la lucha en defensa y cumplimiento de la cuota de género todavía tiene mucho trecho por recorrer (Albaine, 2014: 3).

En 2012, la Comisión Económica para América Latina y el Caribe (CEPAL) establecía que, respecto del número de mujeres en el Poder Legislativo, Cuba iba a la cabeza con el $45.2 \%$; en segundo lugar se encontraba Nicaragua con el 40.2\%, y en tercero Costa Rica con el 38.6\%; Estados Unidos tenía sólo el 16.6\%. Los países con menos mujeres legisladoras eran Panamá, con un
8.4\%, Haití con un 4.2\% y Belice con un 3.1\% (Galeana, 2012: 19)

En México, en los años ochenta y noventa del siglo $\mathrm{XX}$ se promovieron algunas iniciativas para mejorar la condición de las mujeres en el ámbito político:

Algunos partidos liberales y de izquierda han propuesto candidatas feministas en elecciones nacionales, estatales y municipales, y presentan en sus plataformas y programas postulados de claro contenido feminista; reformas de leyes y reglamentos discriminatorios, despenalización del aborto, creación de organismos estatales de coordinación de servicios para las mujeres [...] etc. (De Barbieri y Oliveira, 1986: 15).

El origen de la cuota de género en México data de 1993, cuando en el Código Federal de Instituciones y Procedimientos Electorales (COFIPE) se señaló que los partidos políticos debían promover la participación de la mujer en la vida pública; pero fue en 1996 cuando se estableció que "en las candidaturas de diputados y senadores no habrá más del 70\% de candidaturas del mismo sexo; situación que se vuelve obligatoria en 2002" (Guzmán y Moreno, 2007: 19). No obstante, continuó la resistencia por parte de algunos dirigentes de los partidos políticos, quienes obstaculizaban alcanzar la equidad de género en las instituciones políticas de representación con prácticas políticas indebidas y una cultura política patriarcal y de subordinación.

En el proceso electoral federal de 2015 los líderes de los partidos políticos desplegaron acciones tendientes a cumplir "formalmente" con la obligatoriedad legal de postular candidatos y candidatas, bajo el principio de paridad de género, sin que ello garantizara la representación equilibrada entre mujeres y varones en los órganos de gobierno y legislativos.

En cuanto a la participación de las mujeres en el Congreso Federal, es importante subrayar que el crecimiento es cada vez mayor. En la Cámara de Diputados, en el periodo 2012-2015 las mujeres representaban el 37\%, mientras que en la Cámara de Senadores constituían el 33\% (ADN Político, 2013); un 
poco más de un tercio del Congreso estaba integrado por mujeres - cerca del 40\%-. Se debe subrayar que, en la norma inmediata anterior (2008), "la ley de cuotas establecía una proporción de 60/40 de candidaturas de género para la cámara baja y el Senado" (Palma y Chimal, 2011: 53).

Por ello, la reforma electoral de 2014 significó un gran avance legal debido a que elevaba al 50\% el porcentaje para inscribir candidaturas de un solo sexo, además de que los partidos debían proponer en cada fórmula candidatos propietario y suplente del mismo género, y en la listas plurinominales deberían de alternarse varones y mujeres para garantizar el equilibrio de géneros en las representación política.

Sin embargo, las elecciones federales de 2015 presentaron claroscuros en cuanto al cumplimiento y efectividad de la cuota de género, pues no se logró incrementar significativamente la presencia de mujeres en la representación política. Sólo en algunos casos locales, como en Tabasco, se logró la paridad de género en el Congreso Estatal, por lo que ésta se convirtió en la entidad a la vanguardia en la materia.

A nivel federal, el número de distritos ganados por mujeres aumentó en cifras absolutas: en 2012 ganaron 89 de los 300, y en 2015 obtuvieron 117. Se observó un incremento de veintiocho diputaciones y nueve puntos porcentuales más que en la legislatura anterior; los hombres ganaron en 183 distritos electorales, lo que significa un $61 \%$ de varones y un $39 \%$ de mujeres (INMUJERES, 2015a). En cuanto a la asignación de diputadas por representación proporcional, en 2015 fueron otorgadas 95 diputaciones plurinominales a mujeres, un $34.5 \%$ de las 200 curules repartidas, misma cifra que en las elecciones de 2012 (ver cuadro 1).

En total las mujeres obtuvieron 212 diputaciones por ambos principios, que dan un total de 42.4\%; sólo un 5\% más. Aquí valdría preguntar: ¿qué pasó en 2015 con la paridad de género? Si esta vez se obligó a los partidos políticos a postular a sus candidatos en la misma proporción de hombres y mujeres, si se cuidó que en la misma fórmula fueran candidatos del mismo sexo y si en las listas de representación proporcional figuraba un orden aleatorio entre sexos, ipor qué el porcentaje de diputadas (42.4\%) se colocó en un 8\% de distancia del mínimo esperado?

A nivel de los estados y localidades en donde hubo elecciones, la situación se repitió casi invariablemente, como veremos en este apartado a propósito de lo que ocurrió en Tabasco, el Estado de México y el Distrito Federal. Dicho contexto confirma que no basta con el precepto legal para que, en los hechos, se alcance el equilibrio en la representación política, condición imprescindible para alcanzar una democracia sustantiva en México.

Los partidos políticos se escudaron en el "cumplimiento en sentido vertical" —alternación en las listas y fórmulas - para evadir la inclusión del género en lo horizontal — paridad en número de mujeres y hombres encabezando planillas municipales o delegaciones-; hubo un claro incumplimiento en la dimensión sustantiva o material, que implica colocar a mujeres en aquellos distritos o ayuntamientos en donde el partido político tiene probabilidades de ganar; y que algunas autoridades electorales fallaron en la aplicación de la "trascendencia de género" en la asignación de curules por el principio de representación proporcional.

La dimensión sustantiva en materia de género se refiere a "la falta de sanciones en caso de incumplimiento y mecanismos de monitoreo de estas leyes, así como la interpretación desfavorable hacía las mujeres de dichas leyes por las autoridades judiciales o electorales" (CIDH, 201l: 54).

Mientras que la "trascendencia del género" tiene que ver con la interpretación positiva de la ley, en el sentido de que "[...la] normativa hace extensiva la cuota de género a las candidaturas por principio de mayoría relativa y de representación proporcional, estableciendo que el porcentaje de cuotas debe cumplirse para las candidaturas propietarias y suplentes" (Reynoso y D’Angelo, 2012: 294).

En un estudio sobre el impacto del sistema de cuotas, Esperanza Palma y Abraham Chimal enfatizan que: "La eficacia de las cuotas depende de ciertas características del sistema electoral y de las sanciones por el incumplimiento, así como del mandato de posicionamiento en las listas" (Palma y Chimal, 2011: 
56). De esta manera, el impacto se registra en la norma, pero también en la aplicación, vigilancia y efectividad.

Por ello, la Comisión Interamericana de Derechos Humanos recomienda implementar "medidas necesarias - de naturaleza legislativa, política y regulatoriapara remover los obstáculos estructurales y formales que enfrentan las mujeres en el acceso igualitario a los puestos de toma de decisiones (CIDH, 2011: 75).

En el contexto de las elecciones federales y locales de 2015, en las que se aplicó la recién aprobada Ley de Cuota de Género bajo el principio de paridad, destaca lo ocurrido en el estado sureño de Tabasco, en el cual se logró la paridad de género en la integración del Congreso local, a pesar de las resistencias y malas prácticas de líderes de los partidos políticos de la entidad. Para entender la singularidad e importancia de este hecho, se contrasta con lo que ocurrió en el Estado de México y en el Distrito Federal, dos entidades del centro del país, que en dicha materia de efectividad de la paridad de género caminaron por senderos distintos.

Por ello, el objetivo de este artículo es destacar que, en la implementación y efectividad de la cuota de género en México, hay tres factores presentes que nos explican la baja efectividad de la medida: 1) resistencia de los partidos políticos a acatar la cuota de paridad de género; 2) la práctica partidista de no acatar la dimensión sustantiva o material, al colocar a mujeres en distritos y ayuntamientos - delegaciones - con pocas o nulas probabilidad de ganar, y 3 ) problemas de interpretación de las autoridades electorales al aplicar la trascendencia de género en la asignación de escaños por la vía plurinominal.

El trabajo está dividido en tres apartados. En primer lugar se presentan las bases conceptuales de la representación descriptiva, argumento que fundamenta la necesidad de aplicar la cuota de género como una medida transitoria y correctiva de la situación de exclusión política de la mujer. En segundo lugar se analiza la participación política de las mujeres en Tabasco, estado en el que destaca la penosa situación de "las juanitas" en las elecciones municipales y el papel de avanzada del órgano electoral local, al aplicar la trascendencia de género en la asignación de diputaciones plurinominales. Por último, en forma comparativa y con el objetivo de contrastar el caso de Tabasco, se revisa lo que ocurrió en el Estado de México y en el Distrito Federal, tanto en las elecciones municipales como en las de diputados locales.

\section{Representación política y cuota de género}

La cuota de género es una acción afirmativa que busca remediar una situación de discriminación y de subrepresentación de la que es objeto la mujer frente al varón. Es una norma contenida en la Constitución y en las leyes electorales de los países en donde, históricamente, las mujeres han sido sistemáticamente excluidas de los espacios de poder bajo el argumento - equivocado - de que es una actividad propia de los hombres. En los hechos, la cuota de género busca que los partidos políticos postulen a candidatos de ambos sexos en una situación de equilibrio, tanto como las mismas características de la sociedad lo determinen.

Desde la teoría de la representación política, la tradición liberal no está muy de acuerdo con hacer distinción entre géneros o condiciones socioeconómicas bajo el argumento de que en los "pactos sociales fundacionales" de las democracias (Pescader, 2006: 24) se instituyó la idea de que "un representante actúa en nombre de otros, en su interés o como su agente" (Pitkin, 1985: 18-19). De esta forma, independientemente de las diferencias sociales, de género, raza u otro tipo, un representante es un representante de todos -llámese gobernante, alcalde, diputado o senador - porque actúa por el bien común y los ciudadanos - formalmentehan delegado su representación en éste. Dicha idea, que parte de considerar la sociedad como homogénea, supone que esa es la forma de llegar a una representación sustantiva mediante la que se gobierna y legisla por el bien general sin importar las diferencias étnicas, sociales o económicas: "para el gobierno de casi cualquier institución es preferible un órgano representativo frente a otro que no lo sea (Lifanti, 2009: 500, cursivas añadidas).

Desde una postura más sociológica y progresista, menos liberal y de la tradición jurídica, consideramos que en las naciones diversas, en donde hay diferencias 
evidentes por cuestiones étnicas, de género, edad o condición sociocultural, y hay una larga historia de marginación y exclusión, es válido retomar la idea de la "representación descriptiva", en tanto que grupos de jóvenes, mujeres, indígenas o negros no están o no se sienten representados por políticos y partidos políticos que actúan en favor de intereses ajenos a los suyos. Teóricamente la representación descriptiva se basa en contar con una representación que refleje fielmente las características socioculturales de la sociedad, lo que se traduce en un "estar por otros" (Pastor, 2009: 5). La representación así: "suple al representado por medio de una correspondencia de características" (Lifanti, 2009: 501). O bien, como señala Elena García: "La representación se identifica con la existencia de cierta correspondencia entre representantes y representados, que compartirán determinadas características o cualidades (García, 2011: 206).

Hanna Fenichel Pitkin señala que la representación descriptiva descansa en la idea de que un cuerpo representativo tiene una semejanza con aquello que representa, de tal forma que:

La verdadera representación exige que el legislativo sea elegido de una forma tan estricta que su composición corresponda con toda exactitud a la de toda la nación, sólo entonces será verdaderamente un cuerpo representativo. John Adams arguye que en el periodo revolucionario americano, un legislativo representativo "debería ser un retrato exacto, en miniatura, del pueblo en toda su amplitud, y que debería pensar, sentir, razonar y actuar como este último" (Pitkin, 1985: 65).

Dicha concepción tiene mayor sentido en sociedades en las que hay una crisis de representación en su sentido liberal porque políticos, autoridades, partidos e instituciones públicas no cuentan con la confianza ciudadana; en sociedades en las que la representación política se ha convertido en defensora de intereses de grupo, alejadas del bien común; y en países en donde hay sectores sociales, étnicos o culturales que históricamente han sido marginados y excluidos. Es en dichas naciones en donde se requiere de una representación que refleje la variedad de intereses que existen en la sociedad. En tal sentido, un gobierno representativo debe reflejar la diversidad social y poblacional de su colectividad.

Se respeta la postura de aquellos que consideran que existe una única representación política, aquella emergida de la postulación de candidatos de partidos políticos que "nos representan a todos" más allá de la diversidad social, cultural y étnica de la sociedad. Pero aquí consideramos que no puede existir una representación sustantiva sí en los hechos no hay primero una representación descriptiva, y más si hay sectores sociales, étnicos o culturales - mujeres, indígenas, negros o jóvenes- que históricamente han sido marginados políticamente, o están mal representados o subrepresentados. La idea es que: "la inclusión de los diferentes grupos e identidades de la ciudadanía constituye un aspecto importante para el funcionamiento y la legitimación del régimen democrático representativo" (Reynoso y D`Angelo, 2006: 282).

Las mujeres en México, como en muchos países, han sido históricamente marginadas y excluidas de la actividad política formal, de tal manera que ocupan una situación de minoría política a pesar de representar un poco más del 50\% del total de la población. Alcanzar una democracia sustantiva, a partir primero de una representación descriptiva, tiene mucho que ver con los logros obtenidos a través de las cuotas electorales, por lo que a continuación precisamos dicho término.

El "sistema de cuotas" es una acción afirmativa que posibilita el respeto a los derechos políticos de los ciudadanos y permite alcanzar un mayor equilibrio en la representación política. Lo que se busca con las cuotas electorales para minorías, más que otorgarles un privilegio, es tratar de remediar una situación de desventaja, que en forma transitoria gozarán hasta que se logre el equilibrio.

Dicho sistema de cuotas se utiliza principalmente en sociedades con características diversas en las que minorías sociales, étnicas o culturales son marginadas políticamente. Las más difundidas e instituidas son las "cuotas de género" para mujeres, las "étnicas" para 
indígenas o nativos y las "generacionales" para los jóvenes; aquí sólo nos ocupamos de las primeras.

La cuota de género, como un mecanismo legal que obliga a los partidos políticos a incluir entre sus candidatos a puestos de elección a mujeres, ha tenido un impacto importante para los sistemas democráticos debido a que ha permitido integrar a la diversidad poblacional que conforma una sociedad. A partir del reconocimiento de las mujeres como personas capaces de ocupar puestos públicos de responsabilidad, que poseen perfil profesional, experiencia política y habilidades para desenvolverse en la vida pública, en esa misma medida ha crecido su presencia en la democracia mexicana, garantizando con ello el respeto al derecho político primordial de votar y ser votadas.

Las cuotas, entonces, suponen una mayor centralización del proceso de selección y generan tensiones dentrode los partidosen tanto seenfrentan a la resistencia de aquellos con mayor trayectoria y carrera política. El argumento que debe oponerse es que las cuotas hacen más representativos a los partidos e introducen un concepto distinto de democracia según la cual es necesario crear condiciones de igualdad para el acceso a los cargos públicos (Palma y Chimal, 201l: 58).

Las mujeres han crecido en representación dentro del poder legislativo gracias, entre otras cosas, a la implementación de las "cuotas de género", que han obligado a los partidos a incluirlas como candidatas, tanto en las fórmulas de mayoría relativa como en las listas de representación proporcional. No obstante, la efectividad del sistema de cuotas no se remite únicamente al cumplimento legal y formal de la medida, sino a varios factores personales, del contexto social, del sistema político y de la subjetividad — condición de mujer - en el que están insertas (Cedillo, 2014: 3-6).

Dalia Barrera destaca que las mujeres tienen mayores oportunidades de convertirse en candidatas, legisladoras y gobernantes si cuentan con estudios profesionales, tienen experiencia política, han ocupado cargos en la estructura partidista, han sido capaces de establecer vínculos, además de los familiares, con las esferas locales, estatales o federales y sí su condición - subjetiva- de mujer no obstaculiza su desarrollo en la vida política (Barrera, 2003: 2-14). Dichos supuestos veremos que se cumplen en la participación política de las mujeres en Tabasco, el Estado de México y el Distrito Federal.

\section{La participación política de las mujeres en Tabasco}

El Congreso local de Tabasco es unicameral, se renueva cada tres años y está integrado por 35 diputados y diputadas. Para efectos electorales la entidad se divide en veintiún distritos electorales uninominales, en los que se elige un representante propietario y uno suplente por el principio de mayoría relativa. Los restantes catorce escaños se reparten por la vía de la representación proporcional en dos circunscripciones plurinominales.

Las autoridades municipales se integran por un alcalde o presidente municipal, síndicos y regidores o regidoras según el tamaño de las localidades. Tabasco está integrado por diecisiete municipios, que son: Balancán, Cárdenas, Centla, Centro, Comalcalco, Cunduacán, Emiliano Zapata, Huimanguillo, Jalapa, Jalpa de Méndez, Jonuta, Macuspana, Nacajuca, Paraíso, Tacotalpa, Teapa y Tenosique. De éstos, Huimanguillo es la localidad más extensa y Paraíso la más pequeña; en cuanto a población, el Centro es el que reporta más número de habitantes, y Emiliano Zapata el que menos. Tabasco cuenta con 2238603 habitantes, en el Centro hay 640359 habitantes y en Emiliano Zapata sólo 29518 (INEGI, 2010).

En materia de representación política de las mujeres en Tabasco, ya se señaló que la presencia de éstas en la gubernatura ha sido nula; en las presidencias municipales es mínima, pues en 2012 sólo gobernaban una alcaldía y en 2015 lograron cuatro de las diecisiete, un 23.5\%; sólo en el Congreso local hay una presencia considerable, pues en 2012 representaban un poco más del 40\%. Por tal motivo, en las elecciones locales de 2015, cuando se renovarían las 35 diputaciones locales y los 17 ayuntamientos, bajo las nuevas disposiciones 
legales de "cuota de paridad de género" se pensaba que la participación femenina, en cantidad y calidad, aumentaría considerablemente.

Las consecuencias del proceso electoral local en Tabasco (2015) en materia de género nos arrojan una doble vertiente: una negativa y otra positiva. La primera tuvo que ver con los comicios municipales, que estuvieron manchados por el fenómeno de "las juanitas" y se apreció un avance cuantitativo mínimo. La positiva tuvo que ver con la conquista de la paridad de género en la integración del Congreso local gracias a la aplicación de la "trascendencia de género" en la asignación de escaños de representación proporcional.

En las elecciones municipales de Tabasco en 2015 hubo una resistencia de los partidos políticos a acatar la cuota de género, pues los principales, el Partido Revolucionario Institucional (PRI) y el Partido de la Revolución Democrática (PRD), decidieron no respetar la obligación de proponer candidaturas bajo el principio de paridad de género en forma horizontal, sólo en forma vertical. Ello significaba colocar en la mayoría de las planillas como alcalde a un varón, y alternar el género en forma descendente, en sindicaturas y regidurías; pero no propusieron el 50\% de candidatas a alcaldes, es decir, que ocho mujeres encabezaran por lo menos ocho planillas municipales.

Clave del asunto fue que, a finales de abril de 2015, por orden del Tribunal Electoral del Poder Judicial de la Federación (TEPJF), los partidos políticos PRI y PRD tuvieron que sustituir a candidatos varones con el objetivo de cumplir con la cuota de género en forma horizontal, que los obligaba a postular ocho candidatas en alcaldías, en vez de una que propusieron originalmente. La simulación ocurrió cuando, en vez de recurrir a sus bases y estructura partidista para seleccionar a las mujeres que sustituirían a los hombres, éstos decidieron colocar en su lugar a esposas, hermanas e hijas de los políticos impugnados (Diego, 2015: 1). Los partidos políticos respondieron con el nepotismo para cumplir formalmente con la paridad de género.

En el cuadro 2 se registra el lazo de parentesco que tenían las candidatas que sustituyeron a los varones, en donde se destaca que, de las ocho mujeres que reemplazaron a candidatos impugnados, seis eran esposas de éstos, una hija del presidente municipal en funciones, y una más hermana del candidato. Al final del proceso, tres de las mujeres señaladas como "juanitas" resultaron candidatas ganadoras: Esperanza Méndez Vázquez, Ana Lilia Díaz Zubizarreta y Gabriela López Sanlucas. Sólo Manuela del Pilar Ríos López, quien ganó por el Partido Verde Ecologista de México (PVEM) en Emiliano Zapata, no fue señalada como "juanita" (ver cuadro 2).

El contexto de los resultados de la elección municipal en Tabasco nos indica que el número de alcaldías aumentó de una a cuatro, pasando del 6\% al $23.5 \%$, un avance considerable aunque lejano al $50 \%$ esperado, dado que se obligó a todos los partidos políticos a postular al menos a ocho mujeres.

Por partidos políticos, dos candidatas ganadoras fueron postuladas por el PRD-Nueva Alianza, una por el PRI y otra por el PVEM (ver cuadro 3). Cuantitativamente el avance fue mínimo y, cualitativamente, las prácticas a que recurrieron los partidos políticos para postular a sus candidatas dejaron de lado cualquier evaluación sobre los méritos políticos o profesionales que éstas poseían para ocupar dicho cargo.

Lo rescatable del caso Tabasco, en materia de género, está en lo que ocurrió en la integración del Congreso local, en donde se alcanzó la paridad de género al obtener diecisiete escaños para mujeres y dieciocho para varones (ver cuadro 4). La representación política estaba partida prácticamente por mitades y la entidad se constituye como una de las primeras en alcanzar un equilibrio en la representación legislativa en México.

En realidad, las mujeres en Tabasco tuvieron un mal papel en la competitividad por ganar los distritos, pues sólo se alzaron con el triunfo en seis de los veintiún distritos electorales que integran la entidad: el 28.5\% del total. Tres escaños de mayoría fueron para mujeres propuestas por el PRD, dos por el PRI y una por MORENA (ver cuadro 5).

El equilibrio en la representación femenina en el Congreso de Tabasco tuvo que ver con el proceso de asignación de curules por representación proporcional 
que efectuó el Instituto Electoral y de Participación Ciudadana de Tabasco (IEPCT), organismo que, en un hecho insólito, asignó once diputaciones a mujeres y sólo tres a hombres. Como el estado se divide en dos circunscripciones, decidió asignar a cinco mujeres en la primera y a seis en la segunda para "compensar" de alguna manera la representación política y alcanzar la paridad de género en la legislatura local (ver cuadro 6).

El IEPCT decidió, con el argumento de la trascendencia de género en la asignación de diputados y diputadas por la vía plurinominal, que en la integración del Congreso debía aplicar la paridad de género, considerando ambos principios de elección. Por este motivo, Tabasco se convirtió en una de las entidades a la vanguardia en cuanto a representación política femenina en situación de equidad y paridad, como ninguna otra entidad, como veremos con los casos del Estado de México y el Distrito Federal.

\section{Los casos del Estado de México y el Distrito Federal}

Para evaluar la aplicación de la cuota de paridad de género en Tabasco y qué tan sorprendente fue la decisión del órgano electoral local al garantizar la equidad en la representación política en su recinto legislativo, a continuación analizaremos lo que sucedió en el Estado de México y en el Distrito Federal en 2015, primero en las elecciones municipales o de delegaciones y luego en la integración del Congreso mexiquense y en la Asamblea de Representantes del Distrito Federal.

En las elecciones municipales del Estado de México sólo se obligó a los partidos a cumplir con la paridad de género en forma vertical, pero no horizontal, pese a los recursos de impugnación. El TEPJF resolvió que la obligatoriedad se debía aplicar hasta el siguiente proceso electoral local; por ello, no se exigió a los partidos políticos postular un mínimo de 62 mujeres como candidatas a alcaldesas. La entidad se integra por 125 municipios.

En contraste, similar a lo que ocurrió en Tabasco, en el Distrito Federal se obligó a los partidos políticos, por orden del Tribunal Electoral, a postular candidatas en al menos ocho de las dieciséis delegaciones que integran la capital del país; se observó además la dimensión sustantiva o material, pues deberían colocar a mujeres como candidatas en aquellas delegaciones en donde los partidos políticos tenían posibilidades reales de ganar. No obstante, tanto en el Estado de México como en el Distrito Federal, el aumento de la participación de mujeres como presidentas municipales o jefas delegacionales fue exiguo.

En la elección de presidentes municipales en el Estado de México de 2015, pese a la resistencia de los partidos políticos por postular al menos un 50\% de candidatas mujeres, sí hubo un incremento en el número de alcaldesas, siete más que en la elección de 2012. El porcentaje creció del $11.20 \%$ al 16.8\% (ver cuadro 7 ), suma que coloca a la entidad mexiquense a la vanguardia en cantidad de presidentas municipales.

No obstante, el porcentaje de mujeres que se alzaron con el triunfo en los 125 ayuntamientos mexiquenses es todavía lejano al esperado $50 \%$. Un factor de la baja tasa de efectividad es la colocación de mujeres en postulaciones por alcaldías con pocas probabilidades de obtener el triunfo; además, en fuentes periodísticas se documentó que muchas de las candidatas eran hijas o familiares de políticos miembros de la élite local, como en el caso de la priista Aurora Denisse Ugalde Alegría, hija de Francisco Ugalde. De manera similar a lo que ocurrió en Tabasco, al final no importan las cualidades personales o profesionales de las mujeres ganadoras, sino los prejuicios - reales o falsos- construidos en torno a su postulación.

De las veintiún mujeres presidentas municipales en la entidad mexiquense, se debe subrayar que diecisiete fueron abanderadas por el PRI, tres por el PAN y una por Nueva Alianza; además, se debe subrayar que fue en cuatro municipios urbanos, densamente poblados y desarrollados donde se alzó con el triunfo alguna mujer: Atizapán de Zaragoza, Cuautitlán, Chimalhuacán y Tlalnepantla (ver cuadro 8).

En el Distrito Federal, a pesar de la normatividad relacionada con la cuota de género, bajo el principio de equidad, y la lucha legal de algunas mujeres para que los partidos políticos respetaran el derecho a ser postuladas 
en número, pero también con posibilidad de triunfo, los resultados no fueron los esperados, pues las mujeres ganaron en apenas cuatro de las dieciséis delegaciones (ver cuadro 9).

En el periodo 2012-2015 ocuparon una jefatura delegacional cinco mujeres, las cuales fueron: Lic. Nora del Carmen Bárbara Arias Contreras, en la delegación Gustavo A. Madero; C. Elizabeth Mateos Fernández, en Iztacalco; Lic. Leticia Quezada Contreras, en la delegación Magdalena Contreras; C. Angelina Méndez Álvarez, en Tláhuac, y C. Marisela Contreras Julián, en Tlalpan (INMUJERES, 2015). Ellas representaban un $31.2 \%$ del total de delegados en la capital del país, un tercio de las dieciséis delegaciones.

En 2015 el número de delegadas bajó a cuatro, un $25 \%$ del total. Sólo en una delegación repitió una mujer como delegada, el caso de Tlalpan, con Claudia Sheinbaum Pardo. En cuanto a filiación partidista, dos son del PRD, una del PAN y otra de MORENA; en tamaño y jerarquía de las delegaciones, las cuatro son de singular importancia en la capital (ver cuadro 10).

Parece que en el Distrito Federal, como en las otras dos entidades que nos ocupan, el problema no está tanto en que los partidos políticos cumplan al postular un número determinado de mujeres para ocupar un cargo público y así cumplir con la cuota de género, sino en lo que se conoce como la dimensión material o sustantiva de dicho deber, que implica definir las posibilidades de que éstas tengan probabilidades reales de ganar. Señalan Palma y Chimal que: "una de las conclusiones fundamentales de la literatura comparada es que una legislación que obligue a los partidos a incluir cuotas en las candidaturas hace una enorme diferencia para el resultado final en la composición de su congreso" (Palma y Chimal, 201l: 56).

En cuanto a la integración de las legislaturas locales, en el Estado de México las mujeres duplicaron el número y porcentaje de curules obtenidos anteriormente, pero lejos del esperado $50 \%$ que marca la paridad de géneros (ver cuadro 11). Ganaron 19 de 45 distritos electorales, un $42 \%$ del total, lo cual indica que las mujeres en el terreno de la representación han avanzado en forma sustancial.
La Asamblea de Representantes del D.F. se integra por 66 legisladores, de los cuales en el periodo 2012-2015 un total de 22 eran mujeres y 44 hombres; las mujeres apenas representaban el 33.3\%. Para la elección de 2015 fueron electas un total de 28 mujeres, que dan un total de $42.42 \%$, como se ve en el cuadro 12. La representación de este género creció en un $9 \%$, pero todavía lejos de las 33 diputadas locales que se pretendían alcanzar con la implementación de la paridad de género promovida por la reforma electoral de 2014 (ver cuadro 12).

En cuanto a la aplicación de la "trascendencia de género" en la asignación de diputados y diputadas de representación proporcional, en el Estado de México no se empleó; se aplicó la ley a rajatabla sin importar el género. Por su parte, en el Distrito Federal sí se hizo, pero no se trató de compensar con curules plurinominales a mujeres que no habían ganado en los distritos electorales, bajo el principio de mayoría relativa, como ocurrió en Tabasco.

En el Estado de México se reparten treinta curules de representación proporcional, que sumados a los 45 de mayoría relativa dan los 75 espacios en el Congreso local. En dicha entidad se ignoró completamente el género en el reparto de curules por el principio de representación proporcional, pues de los treinta escaños que se otorgan por este principio sólo nueve fueron para mujeres, un $30 \%$, y veintiuno para varones, el $70 \%$.

Por principio, se debe señalar que, de los siete escaños asignados al PAN, sólo dos fueron para mujeres; de los seis del PRD, también dos para mujeres; de cinco que obtuvo MORENA, sólo dos fueron para mujeres; Movimiento Ciudadano y el Partido Encuentro Social (PES) colocaron una de tres; Nueva Alianza una de dos, y el PT y PVEM ninguna. El PRI no tuvo acceso a la representación proporcional. En las asignaciones realizadas por el Instituto Electoral del Estado de México (IEEM) no se reflejó el espíritu de la paridad de género.

Resulta que, salvo el PES, todos los demás partidos políticos no colocaron a una mujer en el primer lugar de la lista de diputados plurinominales de representación proporcional, por lo que sus posibilidades de asegurar un sitio en la Cámara de Diputados local disminuyó. 
En la capital del país, por su parte, desde fines de abril de 2015 el Instituto Electoral del Distrito Federal (IEDF) comunicó a los partidos políticos un acuerdo mediante el cual les advertía de que, en la asignación de asambleístas por la vía plurinominal, aplicaría la trascendencia de género. De esta forma buscaría que en la repartición de curules se diera lo más fiel posible la paridad de género. Por ello, de los veintiséis escaños repartidos, catorce correspondieron a varones y doce a mujeres, el $54 \%$ y el $46 \%$ respectivamente. De los cinco escaños del PAN, dos fueron para mujeres; de los cuatro del PRI, otros dos; de los siete del PRD, cuatro; de los dos del PVEM, uno fue para una mujer; de los cuatro de MORENA, dos; de los dos del PES, una mujer, y Movimiento Ciudadano y el Partido Humanista lograron una curul cada uno, varones ambos. La igualdad no se logró porque invariablemente los partidos políticos colocaron a varones en el primer lugar de las listas.

Lo que se debe destacar aquí es la denuncia que interpusieron organizaciones y mujeres, que acusaron al IEDF de no apegarse al principio de paridad de género a la hora de aplicar la asignación de diputadas plurinominales, pues lo que se buscaba era que el IEDF compensara por dicho principio lo que las mujeres no habían logrado en los distritos (Melgar, 2015: 1).

$\mathrm{El}$ argumento es que, si existe el mecanismo de compensación, que es la asignación de diputados por representación proporcional, como se dio en Tabasco, había que considerarlo, ya que con ello se lograría reflejar el espíritu de la ley y también, de paso, evitar el problema de la dimensión sustancial o material que invoca la postulación de mujeres en distritos en donde no tienen muchas posibilidades de éxito, y que los partidos políticos no reserven para los varones aquellos en los que tienen más probabilidades de ganar.

\section{Conclusiones}

En el proceso electoral federal de 2015, en el cual se estrenó la reforma política de 2014, que entre otras modificaciones establecía la cuota de género en situación de paridad, 50\% para hombres y 50\% para mujeres, en todos los cargos y por ambos principios de elección - mayoría relativa y representación proporcional-, estaban echadas todas las cartas para lograr una representación más equilibrada justa e incluyente, situación que en los hechos no ocurrió.

En Tabasco, ante la obligación para los partidos políticos de cumplir con la cuota de género, éstos recurrieron a prácticas ligadas al nepotismo, ya que en la postulación o sustitución de candidatos impugnados ante el TEPJF rellenaron las listas y fórmulas con familiares - esposas, hermanas o hijas - de los políticos varones. De esta forma, colocaron a "juanitas", en vez de recurrir a la base social y partidista, cuadros de mujeres dentro de los partidos políticos, para postular a candidatas de calidad, con amplia experiencia y trayectoria política para ganar los puestos de elección.

Afortunadamente, grupos de mujeres, candidatas excluidas y dirigentes de los mismos partidos dieron la lucha y denunciaron las acciones de las dirigencias partidistas, de los consejeros electorales en los institutos electorales locales, y de muchos políticos que se negaban en los hechos a cumplir con el mandato legal de paridad de género. En Tabasco, el Distrito Federal y el Estado de México se interpusieron recursos legales para obligar a los partidos políticos a respetar la cuota de género; ante esta situación, los institutos electorales de Tabasco y de la capital del país rectificaron, no así el del Estado de México, como se señaló en su momento.

Encuantoal sistema políticoy alos procedimientos de asignación de cargos, es revelador que en la distribución de escaños por representación proporcional, en varias entidades y espacios de gobierno no se recurrió al espíritu de la ley de garantizar la paridad de género, salvo el caso de Tabasco, en donde a través de las plurinominales se alcanzó la paridad de género. En el Distrito Federal la situación fue de apego a la ley, pues se repartió en paridad de género, independientemente de lo ganado por mayoría relativa; en el Estado de México no se cumplió, pues de los treinta escaños sólo nueve fueron para mujeres.

Lo ocurrido en las tres entidades analizadas en 2015 deja como enseñanza que, en la lucha por alcanzar la paridad de género, las mujeres deben ser 
incluidas en la postulación de cargos en los sentidos vertical y horizontal, de modo que se debe garantizar la dimensión sustancial o material en los distritos o ayuntamientos en donde son postuladas. y en la asignación de curules de representación proporcional se debe aplicar la trascendencia del género, como lo mandata la reforma electoral de 2014. Sólo de esa manera se logrará la pretendida equidad de género en México.

\section{Notas}

1 "Juanitas" es un sobrenonmbre que refiere a una práctica política en México que consiste en simular candidaturas de mujeres que, en caso de ganar, renuncian al cargo para dejar que asuma el suplente varón, quien generalmente es el esposo o un familiar de la candidata. El nombre surgió de un político llamado Juan, que se prestó a tal simulación en la delegación Iztapalapa en el Distrito Federal, ciudad capital de México.

\section{Referencias bibliográficas}

Albaine, Laura (2014). "Paridad de género y violencia política. Los casos de Bolivia, Costa Rica y Ecuador". Ponencia presentada en el XXV Congreso Nacional y $\mathrm{V}$ Internacional de Estudios Electorales. Integridad y Equidad Electoral en América Latina. San José, Costa Rica, 11 al 14 de noviembre.

Barrera Bassols, Dalia (2003). "Presidentas municipales en México. Perfiles y trayectorias”. En Cuicuilco, vol. 10, núm. 27, enero-abril. México: Escuela Nacional de Antropología e Historia.

Carbonell, Miguel (2015). Ley General de Instituciones y Procedimientos Electorales. México: Porrúa.

Cedillo Delgado, Rafael (2014). "El perfil profesional de las presidentas municipales del Estado de México: 2009-2012". Ponencia presentada en el Segundo Congreso Internacional de Ciencia Política. Asociación Mexicana de Ciencia Política, Toluca México, 10 al 13 de septiembre.

CIDH (Comisión Interamericana de Derechos Humanos) (2011). El camino hacia una democracia sustantiva; la participación política de las mujeres en las Américas. Finlandia: CIDH y OEA.

De Barbieri, Teresita y Orlandina de Oliveira (1986) "Nuevos sujetos sociales: la presencia política de las mujeres en América Latina”. En Nueva Antropología, vol. VIII, núm. 30. México.

Diego, Juan Manuel (2015). "PRI y PRD defienden postular 'Juanitas' en Tabasco”. En El Universal en línea, México, 30 de abril. Disponible en: http://www.eluniversal. com.mx/estados/2015/defienden-a-juanitas-1096612. html (consultado en mayo de 2015).

Galeana, Patricia (2012). "Historia comparada de las mujeres en las Américas". En Patricia Galeana (coord.), Historia comparada de las mujeres en las Américas. México: Instituto Panamericano de Geografía e Historia-UNAM.

García Guitián, Elena (2011). "Crisis de la representatividad política: las exigencias de la política de la representación”. En Revista de Estudios Políticos (Nueva Época), núm. 1ll, enero-marzo. México.

Guzmán, Virginia y Claudia Moreno (2007). Hacía un horizonte paritario en América Latina: representación política de las mujeres. Documento presentado en la Décima Conferencia Regional sobre la Mujer de América Latina y el Caribe. Quito, Ecuador: CEPAL.

INEGI (Instituto Nacional de Estadística, Geografía e Informática) (2010). Censo de Población y Vivienda 2010. México: INEGI. Disponible en: http://www3. inegi.org.mx/sistemas/TabuladosBasicos/Default. aspx?c=27302\&s=est (consultado en mayo de 2015).

INMUJERES (Instituto Nacional de las Mujeres) (2015). "Observatorio de la Participación Política de las Mujeres en México". México: INMUJERES. Disponible en: http://oppmm.rigter.ninja/mapa/ (consultado el 28 de junio de 2015).

INE (Instituto Nacional Electoral) (2013). "Cámara de Diputados". En INE, Género, igualdad y democracia. México: INE. Disponible en: http://genero.ife.org. mx/legislativo_dip.html\#diputadosLXII-2013-actual (consultado el 28 de junio de 2015).

IEEM (Instituto Electoral del Estado de México) (2015a). "Diputadas y diputados electos a la LIX Legislatura 
del Estado de México 2015-2018". Toluca, México: IEEM. Disponible en: http://www.ieem.org.mx/2015/ resultados_2015/diputados2015.pdf (consultado el 28 de junio de 2015).

IEEM (Instituto Electoral del Estado de México) (2015b). "Diputadas y diputados electos a la LIX Legislatura del Estado de México 2015-2018". Toluca, México: IEEM. Disponible en: http://www. ieem.org.mx/2015/resultados_2015/Ayuntamientos/ PlanillasGanadoras2015_18062015.pdf (consultado el 28 de junio de 2015).

Instituto Electoral y de Participación Ciudadana de Tabasco (2015a). "Proceso electoral ordinario 2014 2015”. México: IEPCT. Disponible en: http://iepct. org.mx/resultados_elecciones_2015/resultados.htm (consultado el 28 de junio de 2015).

Instituto Electoral y de Participación Ciudadana de Tabasco (2015b). "Entrega constancia de mayoría de diputados y regidores plurinominales". México: IEPCT. Disponible en: http://www.iepct.org $\mathrm{mx} /$ docs/boletines/20150615_\%20B000164.pdf (consultado el 28 de junio de 2015).

Lifante Vidal, Isabel (2009). "Sobre el Concepto de Representación”. En DOXA. Cuadernos de Filosofía del Derecho, núm. 32. España: Universidad de Alicante.

Melgar, Ivonne (2015). "Promueven paridad vía plurinominal en los 32 Congresos. Activistas presentan una queja ante el Tribunal Electoral por falta de equidad de género en las cámaras estatales". En Excélsior, México, 20 de junio. Disponible en: http:// www.excelsior.com.mx/nacional/2015/06/20/1030434 (consultado el 28 de junio de 2015).

Ortiz, Tanía (2015). "Revocan Lista de Candidatos a Diputados del PRD”. En Estadode México al día, 6 de mayo.
Toluca. Disponible en: http://www.edomexaldia.com. $\mathrm{mx} / 2015 / 05 /$ revocan-lista-de-candidatos-a-diputadosdel-prd/ (consultado el 28 de junio de 2015).

Palma, Esperanza y Abraham Chimal (2011). "Partidos y cuotas de género. El impacto de la ley electoral en la representación descriptiva en México". En Revista Mexicana de Estudios Electorales, núm. 1l, julio. México.

Pastor Yuste, Raquel (2009). "Género, representación política y acción parlamentaria: análisis del caso español". Ponencia presentada en el Noveno Congreso de la AECPA, Málaga, 23 al 25 de septiembre.

Pescader, Carlos (2006). La representación política en cuestión. Argentina: Universidad Nacional de la Pampa-Facultad de Ciencias Económicas y Jurídicas. Pitkin, Hanna Fenichel (1985). El concepto de representación. Madrid: Centro de Estudios Constitucionales,

Ramírez, Gabriela (2015). "Ahora al PAN. Revoca TEPJ candidaturas a diputados en el EDOMEX”. EnSeMéxico, 9 de mayo. Disponible en: http://www.semexico.org. mx/archivos/1048 (consultado el 28 de junio de 2015).

Reynoso, Diego y Natalia D’Angelo (2006). "Ley de cuota y su impacto en la elección de mujeres en México". en Políticay Gobierno, vol. XIII, núm. 2, segundo semestre. Ruiz Miguel, Alfonso (1999). "Paridad electoral y cuotas femeninas”. En Claves de Razón Práctica, núm. 94, julioagosto, pp. 48-53.

Torres García, Isabel (2012). "Promoviendo la igualdad: cuotas y paridad en América Latina". En Revista Derecho Electoral, núm. 14, julio-diciembre.

Zepeda, Aurora (2015). “iSabes cuántas mujeres ganaron en las pasadas elecciones?” En Excélsior, 28 de junio. México. Disponible en: http://www.excelsior.com. mx/nacional/2015/06/28/1031788 (consultado el 28 de junio de 2015). 
Cuadro 1. Diputaciones federales para mujeres en 2015, por principio de elección

\begin{tabular}{|c|c|c|c|c|c|c|}
\hline Principio & \multicolumn{2}{|c|}{ Mayoría relativa } & \multicolumn{2}{|c|}{ Representación proporcional } & \multicolumn{2}{|c|}{ Total } \\
\hline Año & \multicolumn{2}{|c|}{$(300)$} & \multicolumn{2}{|c|}{$(200)$} & \multicolumn{2}{|c|}{500} \\
\hline 2012 & 89 & $29.6 \%$ & 95 & $47.5 \%$ & 184 & $36.8 \%$ \\
\hline 2015 & 117 & $39 \%$ & 95 & $47.5 \%$ & 212 & $42.4 \%$ \\
\hline
\end{tabular}

Fuente: elaboración propia con información de INMUJERES (2015: 1).

Cuadro 2. Sustitución de candidatos a alcaldes en Tabasco: 2015

\begin{tabular}{|c|c|c|c|c|}
\hline Partido & Municipio & Candidato & Sustituta & Observación \\
\hline PRD & Jalapa & $\begin{array}{l}\text { Francisco Alfonso } \\
\text { Filigrana Castro }\end{array}$ & Esperanza Méndez Vázquez & Esposa del candidato \\
\hline PRD & Jonutla & Fermín Torres Sánchez & Ana Lilia Díaz Zubizarreta & Esposa del candidato \\
\hline PRD & Centla & Pedro Estrada Córdova & Rosa Icela Hernández & $\begin{array}{l}\text { Hija del alcalde actual } \\
\text { Román Hernández. }\end{array}$ \\
\hline PRI & Jonutla & Erick Garrido & Johana Ricárdez Cabrera & Esposa del candidato \\
\hline PRI & Jalpa & Isidro Peregrino & Lilia del Carmen Gálvez & Esposa del candidato \\
\hline PRI & Centla & Oscar Castillo Moha & Gabriela López Sanlucas & Esposa del candidato \\
\hline PRI & Paraíso & $\begin{array}{l}\text { Carlos Mario de la Cruz } \\
\text { Alejandro }\end{array}$ & Eloísa Guadalupe Ramírez Vidal & Esposa del candidato \\
\hline PRI & Cunduacán & $\begin{array}{l}\text { Francisco Custodio } \\
\text { Gómez }\end{array}$ & Nidia Custodio Gómez & Hermana del candidato \\
\hline
\end{tabular}

Fuente: elaboración propia con información de Diego (2015).

Cuadro 3. Mujeres alcaldesas electas en Tabasco en 2015

\begin{tabular}{lll} 
& \multicolumn{1}{c}{ Municipio } & \multicolumn{1}{c}{ Nombre } \\
\hline Centla & PRI & Gabriela del Carmen López Sanlucas \\
Emiliano Zapata & PVEM & Manuela del Pilar Ríos López \\
Jalapa & PRD-NA & Esperanza Méndez Vázquez \\
Jonutla & PRD-NA & Ana Lilia Díaz Zubieta \\
\hline
\end{tabular}

Fuente: elaboración propia con información de IEPCT (2015b). 


\section{Cuadro 4. Integración del Congreso de Tabasco. 2012-2015 y 2015-2018}

\begin{tabular}{|c|c|c|c|c|c|}
\hline \multicolumn{3}{|c|}{ Curules en el Congreso local 2012-2015 } & \multicolumn{3}{|c|}{ Curules en el Congreso local 2015-2018 } \\
\hline Sexo & Absolutos & $\%$ & Sexo & Absolutos & $\%$ \\
\hline Mujeres & 15 & 42.86 & Mujeres & 17 & 48.57 \\
\hline Hombres & 20 & 57.14 & Hombres & 18 & 51.43 \\
\hline Total & 35 & 100 & Total & 35 & 100 \\
\hline
\end{tabular}

Fuente: elaboración propia información de INMUJERES (2015) e IEPCT (2015a).

\section{Cuadro 5. Diputadas de mayoría relativa en el Congreso de Tabasco 2015-2018}

\begin{tabular}{lll}
\hline \multicolumn{1}{c}{ Distrito } & \multicolumn{1}{c}{ Diputada electa } & \multicolumn{1}{c}{ Partido(s) } \\
I. Tenosique & Yolanda Isabel Bolón Herrera & PRD-NA \\
II. Cárdenas & Zoila Margarita Isidro Pérez & PRI \\
IV. Huimanguillo & María Estela de la Fuente Dagdug & PRD \\
VII. Centro & Yolanda Rueda de la Cruz & PRI \\
XII. Comalcalco & María Luisa Sollemera Corrales & MORENA \\
XX. Paraíso & Ana Luisa Castellanos Hernández & PRD-NA \\
Total: 6 & & \\
\hline
\end{tabular}

Fuente: elaboración propia con información de IEPCT (2015a).

\section{Cuadro 6. Diputados(as) de representación proporcional en Tabasco 2015-2018}

\begin{tabular}{|c|c|c|c|c|c|}
\hline \multicolumn{3}{|c|}{ Primera circunscripción } & \multicolumn{3}{|c|}{ Segunda circunscripción } \\
\hline PAN & Luisa G. Trujillo Rodríguez & & PAN & María Soler Lanz & \\
\hline PRI & Gloria Herrera & in & PRI & Patricia Hernández Calderón & o \\
\hline PVEM & Hilda Santos Padrón & $\ddot{\ddot{d}}$ & PRD & Norma Gamas Fuentes & $\ddot{\ddot{d}}$ \\
\hline MOV.CIU & Teresa Patiño Gómez & $\frac{0}{3}$ & PVEM & Leticia Palacios Caballero & $\frac{\omega}{3}$ \\
\hline MORENA & Guadalupe Bautista Hernández & & $\begin{array}{l}\text { MOV.CIU } \\
\text { MORENA }\end{array}$ & $\begin{array}{l}\text { Manuela Quiroga Mayo } \\
\text { Candelaria Pérez Jiménez }\end{array}$ & \\
\hline PRI & Manuel Andrés Díaz & Hombres: 2 & PRI & José Alberto Lazo Zentella & Hombres: 1 \\
\hline PRD & J. Antonio de la Vega Asmitia & 2010 & & & \\
\hline Total & & 7 & Total & & 7 \\
\hline
\end{tabular}

Fuente: elaboración propia con información de IEPCT (2015b). 
Cuadro 7. Alcaldías por sexo en el Estado de México. 2012-2015 y 2015-2018

\begin{tabular}{rcc|ccc}
\hline & \multicolumn{2}{c|}{ Alcaldía ganadas para $2012-2015$} & \multicolumn{3}{c}{ Alcaldía ganadas para 2015-2018 } \\
\hline Sexo & Absolutos & $\%$ & Sexo & Absolutos & $\%$ \\
Mujeres & 14 & 11.20 & Mujeres & 21 & 16.8 \\
Hombres & 111 & 88.80 & Hombres & 104 & 83.2 \\
Total & 125 & 100 & Total & 125 & 100 \\
\hline
\end{tabular}

Fuente: elaboración propia con información de INMUJERES (2015).

\section{Cuadro 8. Presidentas municipales electas en el Estado de México 2015}

\begin{tabular}{llll} 
No. & \multicolumn{1}{c}{ Municipio } & \multicolumn{1}{c}{ Partido(s) } & \multicolumn{1}{c}{ Candidata electa } \\
\hline 1 & Acambay & PRI & María del Carmen Peña Mercado \\
2 & Aculco & PRI & Aurora González Lezama \\
3 & Atizapan de Zaragoza & PAN-PT & Ana María Balderas Trejo \\
4 & Atlacomulco & PRI-PVEM-NA & Ana María Chimal Velasco \\
5 & Ayapango & PRI-PVEM-NA & María Elizabeth Piedra Bustos \\
6 & Cuautitlán & PRI-PVEM-NA & Martha Elvia Fernández Sánchez \\
7 & Chapa de Mota & PAN-PT & Leticia Zepeda Martínez \\
8 & Chimalhuacan & PRI-PVEM-NA & Rosalba Pineda Ramírez \\
9 & Jiquipilco & PRI & Marisol González Torres \\
10 & Joquicingo & Nueva Alianza & Alma Delia Pallares Castañeda \\
11 & Melchor Ocampo & PAN-PT & Miriam Escalona Piña \\
12 & Mexicalcingo & PRI-PVEM-NA & Sara Vázquez Alatorre \\
13 & Nicolás Romero & PRI-PVEM-NA & Angelina Carreño Mijares \\
14 & Ocoyoacac & PRI-PVEM-NA & Diana Pérez Barragán \\
15 & El Oro & PRI & Cristina Sabina Cruz Hernández \\
16 & Santo Tomás & PRI & Ma. Clotilde García Enríquez \\
17 & Temamatla & PRI-PVEM-NA & Evertina Sánchez Bahena \\
18 & Texcaltitlán & PRI & Zoila Huerta Loza \\
19 & Texcalyacac & PRI & Xóchitl Maribel Ramírez Bermejo \\
20 & Tlalnepantla & PRI-PVEM-NA & Aurora Dennise Ugalde Alegría \\
21 & Tonatico & PRI-PVEM-NA & Ana Cecilia Peralta Cano \\
\hline
\end{tabular}

Fuente: elaboración propia con información del IEEM (2015b). 
Cuadro 9. Delegados y delegadas en el Distrito Federal. Periodos 2012-2015 y 2015-2018

\begin{tabular}{|c|c|c|c|c|c|}
\hline \multicolumn{3}{|c|}{$2012-2015$} & \multicolumn{3}{|c|}{$2015-2018$} \\
\hline Sexo & Absolutos & $\%$ & Sexo & Absolutos & $\%$ \\
\hline Mujeres & 5 & 31.25 & Mujeres & 4 & 25 \\
\hline Hombres & 11 & 68.75 & Hombres & 12 & 75 \\
\hline Total & 16 & 100 & Total & 16 & 100 \\
\hline
\end{tabular}

Fuente: elaboración propia con información de INMUJERES (2015).

\section{Cuadro 10. Mujeres electas en delegaciones del Distrito Federal 2015}

\begin{tabular}{lcc}
\hline \multicolumn{1}{c}{ Candidata } & Delegación & Partido(s) \\
\hline Bertha Xóchitl Gálvez Ruiz & Miguel Hidalgo & PAN \\
Dione Anguiano Flores & Iztapalapa & PRD-PT-NA \\
María Antonieta Hidalgo Torres & Álvaro Obregón & PRD-PT \\
Claudia Sheinbaum Pardo & Tlalpan & MORENA \\
\hline
\end{tabular}

Fuente: elaboración propia con información de INMUJERES (2015).

Cuadro 11. Integración del Congreso del Estado de México. Periodos 2012-2015 y 2015-2018

\begin{tabular}{lcc|ccc}
\hline \multicolumn{2}{c|}{ Curules en el Congreso local 2012-2015 } & \multicolumn{3}{c}{ Curules en el Congreso local 2015-2018 } \\
\hline \multicolumn{1}{c}{ Sexo } & Absolutos & $\%$ & Sexo & Absolutos & $\%$ \\
Mujeres & 13 & 17.33 & Mujeres & 28 & 37.33 \\
Hombres & 62 & 82.67 & Hombres & 47 & 62.66 \\
Total & 75 & 100 & Total & 75 & 100 \\
\hline
\end{tabular}

Fuente: elaboración propia con información de INMUJERES (2015).

Cuadro12. Integración de la Asamblea de Representantes del D.F. Periodos 2012-2015 y 2015-2018

\begin{tabular}{lcc|lcc}
\hline \multicolumn{2}{c|}{ Curules en Asamblea local 2012-2015 } & \multicolumn{3}{c}{ Curules en Asamblea local 2015-2018 } \\
\hline \multicolumn{1}{c}{ Sexo } & Absolutos & $\%$ & Sexo & Absolutos & $\%$ \\
Mujeres & 22 & 33.33 & Mujeres & 28 & 42.42 \\
Hombres & 44 & 66.67 & Hombres & 38 & 57.58 \\
Total & 66 & 100 & Total & 66 & 100 \\
\hline
\end{tabular}

Fuente: elaboración propia, información de INMUJERES (2015) y Melgar (2015). 